\title{
Abstracts from the 1st International Saudi Pediatric Neurology Conference
}

\author{
Sunday, November 23, 2008
}

\section{Epilepsy Symposium: Part I}

\section{Lecture I:}

\section{"Neonatal and Infantile Epileptic Syndromes"} Dr. Perrin Plouin, France

Benign Familial Neonatal Seizures (BFNS) and Benign Idiopathic Neonatal Seizures (BINS) are listed among "idiopathic generalized epilepsies and syndromes" in the International Classification of epilepsies, epileptic syndromes and related disorders. Benign neonatal seizures (BNS) are defined by a favorable outcome, i.e. a normal psycho-motor development and the absence of secondary epilepsy. Many factors among which etiology is the most important take part in the prognosis. However BFNS and BINS fulfill the criteria of BNS even if some questions remain open regarding secondary epilepsy. One can consider the diagnosis of BFNS as a diagnosis by exclusion reinforced by the familial history of neonatal seizures. From the first publications it has been demonstrated an autosomal dominant pattern of inheritance that has been confirmed in all later reports.

BINS were first reported as Fifth's Day Fits in 1977 by Dehan et al. In all cases the convulsions occurred between day (D) 1 and D7, including 90\% between D4 and D6 and 97\% between D3 and D7. When described, the seizures were always of the clonic type, mostly partial, and/or apneic, but never tonic. The mean duration of status epilepticus was about 20 hours Interictal EEG may be normal, discontinuous or in $60 \%$ of the cases the "théta pointu alternant" pattern is present.

\author{
Lecture II: \\ "Epileptic Encephalopathies" \\ Dr. Federico Vigevano, Italy
}

With the term of Epileptic Encephalopathy (EE) we define a "condition in which the epileptiform EEG abnormalities are believed to contribute to progressive disturbance in cerebral function" (ILAE Commission, 2001). In my opinion, more exhaustive is the following definition proposed by Dulac (2001): "Epileptic Encephalopathies are a group of conditions in which cognitive, sensory and/or motor functions deteriorate as a consequence of epileptic activity, which consists of frequent seizures and/or major so called interictal paroxysmal activity".

In this epileptic entity we can identify three conditions:

1. EE mainly due to severe seizures activity: as "Migrating Partial Seizures" or severe partial seizures due to focal cortical dysplasia;

2. EE mainly due to intense EEG epileptiform abnormalities, as in Landau-Kleffner Syndrome or ESES;

3. EE due to severe seizures activity and intense EEG epileptiform abnormalities, as in Ohtahara Syndrome and West Syndrome.

EE usually have a severe prognosis and may look like a progressive disease. Nevertheless, sometimes we can obtain very good results: i.e. remission of spasms with ACTH or VGB; remission of seizures in patients with focal cortical dysplasia or hemimegalencephaly after neurosurgical treatment. Few EE, i.e. ESES, are age-dependent and usually remit spontaneously at puberty. 


\section{Epilepsy Symposium: Part II}

\section{Lecture I:}

\section{"Childhood Epileptic Syndromes" \\ Dr. Colin Ferrie, UK}

In this context the term 'childhood' is taken to mean the period from 2 up to 16 years of age. It is in this age group that the syndromic approach to the epilepsies has been the most helpful and there are now many well recognised syndromes and other proposed syndromes which characteristically begin during it. The International League Against Epilepsy's definition of an epilepsy syndrome is that it is a complex of signs and symptoms that define a unique epilepsy condition. The term should be distinguished from 'epileptic disease' which is a pathologic condition with a single specific, well-defined aetiology. It is because we do not know the underlying aetiologies of most epileptic conditions that we use epilepsy syndromes.

In this lecture the idiopathic generalised epilepsies of childhood and the idiopathic focal epilepsies of childhood will be reviewed. The symptomatic/probably symptomatic focal epilepsies are less age dependent and the syndromic approach has generally been less useful with them.

Childhood and juvenile absence epilepsy and juvenile myoclonic epilepsy, can be considered the 'core' IGEs in children. Epilepsy with myoclonic-astatic seizures (Doose syndrome) and epilepsy with myoclonic absences are now considered as IGEs by the ILAE, although these often behave as epileptic encephalopathies. Other proposed syndromes include eyelid myoclonia with absences, perioral myoclonia with absences and phantom absences. The idiopathic focal epilepsies of childhood include Panayiotopoulos syndrome, benign childhood epilepsy with centrotemporal spikes (rolandic epilepsy) and idiopathic childhood occipital epilepsy (including idiopathic photosensitive occipital lobe epilepsy).

\section{Lecture II:}

\section{"Computed Tomography of the Brain for 1st Seizure in the Emergency Room"}

\section{Dr. Ahmed Al Rumayan, NGH, Saudi Arabia}

The hospital records of 126 children with seizure were reviewed. Patients with known brain tumor, craniotomy, bleeding disorder or open skull fracture were excluded. Type of seizure, physical finding on presentation, developmental delay, other chronic disease, final diagnosis and hospital outcome were cross correlated. Of the 126 patients reviewed, 53(42.1\%) had abnormal CT scan. Abnormal CT scan was significantly associated with the presence of chronic disease $(p<0.01)$, developmental delay ( $p<0.01$ ), physical findings ( $p<$ $0.01)$ and type of seizure $(p=0.04)$. However, adjustment for other variables with the logistic regression analysis showed that; the presence of developmental delay ( $p=0.01$ ), as well as the presence of physical findings $(p<0.02)$ was the only significant predictors of an abnormal CT scan. Children with developmental delay are 5 times more likely to have an abnormal CT $(\mathrm{OR}=4.97)$, while those with focal findings are about 8 times to have an abnormal CT $(\mathrm{OR}=7.85)$ as compared to other children. Our findings show that careful neurological examination as well as history of developmental delay could be considered as significant predictors of an abnormal CT scan in children who present to the emergency department with their first seizure.

\section{Epilepsy Symposium: Part III}

\section{Lecture I:}

"The Panayiotopoulos Syndrome"

Dr. Athanasios Covanis, Greece

Panayiotopoulos syndrome (PS) has been recognized by the ILAEs 2001, 2006 as an early onset benign childhood occipital epilepsy as compared with the late onset childhood occipital epilepsy. It is proposed the alternative name of Panayiotopoulos type for the former and Gastaut type for the latter. During the last decade independent studies from around the world have strongly suggested that these conditions are likely to be separate disorders, rather than variants of a single disorder and that whilst seizures in Gastaut type occipital epilepsy are indeed likely to originate in the occipital lobes, this is unlikely to be the case in PS and therefore, is not an occipital epilepsy.

Autonomic seizures in PS consist of episodes of disturbed autonomic functions with emesis as the predominant symptom. Other autonomic clinical manifestations include pallor, mydriasis, incontinence of urine/or feces, hypersalivation, or cardiorespiratory irregularities. It has high prevalence, affecting around $13 \%$ of children 3-6 years old who have had one or more afebrile seizures and $6 \%$ of such children in the 1-15 year age group. Two thirds of seizures occur during sleep and half of them are prolonged lasting from 30 minutes to several hours. Ictal vomiting occurs in 
the majority of cases but was also reported by Covanis et al 2003, in benign childhood epilepsy with centro temporal spikes. Despite high prevalence, dramatic and lengthy manifestations, PS is often misdiagnosed as nonepileptic conditions. The main reason for this is that emetic and other autonomic manifestations are not recognized as seizure events. PS is remarkably benign despite high incidence of autonomic status epilepticus.

\section{Lecture II: \\ "Childhood Absence Epilepsy" \\ Dr. Yoshimi Sogawa, USA}

Childhood Absence Epilepsy (CAE) is the single most common childhood onset epilepsy syndrome accounting for 10-17\% of all childhood epilepsy. Onset is typically between ages 4 to 8 years and is characterized by very frequent daily absence seizures in an otherwise normal child. The EEG usually demonstrates a $3 \mathrm{~Hz}$ generalized spike-wave discharges, often provoked by hyperventilation. CAE is often considered as an age-specific "benign" epilepsy syndrome because of the favorable response to Anti-epileptic drugs (AEDs) and high remission rate. Partly because of this concept of "benign epilepsy", there have been only a handful of randomized clinical trials and longitudinal prospective studies focused on CAE.

The short term treatment response (2-12 weeks) from the clinical trials has been variable with $50-60 \%$ success rate for the initial AED. This number indicate about half of patients will be tried on the second AED. No prospective randomized clinical trial has documented the long-term clinical response to subsequent AED once initial monotherapy fails. It is known however, that a significant proportion of children who did not respond to initial monotherapy with either ethosuximide or valproic acid respond to the other. Therefore, failure of the initial AEDs in patients with CAE may not indicate poor long-term outcome as in patients with partial epilepsy.

CAE is thought of as a distinct and homogenous "benign" syndrome, when compared to other epilepsy syndrome such as Lennox-Gas taut syndrome. However, the clinical course of CAE is variable and remission is not guaranteed as in Benign Rolandic Epilepsy where the remission rate approaches $100 \%$.

\section{Lecture III:}

"Pregabalin: Preliminary Experience in Intractable Childhood Epilepsy"

Dr. Mohammed Jan, KAUH, Saudi Arabia

Pregabalin, a new antiepileptic drug (AED), is used as adjunctive treatment for partial epilepsy. It acts at presynaptic calcium channels, modulating neurotransmitter release. A prospective, open label, add on trial of pregabalin in treating consecutive children with intractable epilepsy (defined as recurrent seizures after at least 3 antiepileptic medication trials) was carried out. Follow up by one pediatric neurologist was performed. Therapeutic response was recorded as complete (no seizures), Good ( $>50 \%$ seizure reduction), fair $(<50 \%$ seizure reduction), or none.

Nineteen children (63\% males) aged $4-15$ years were included. Most children (74\%) had daily seizures that failed multiple AEDs. The epilepsy was symptomatic in $58 \%$, and $74 \%$ had associated cognitive deficits. The seizures were mixed in $9(47 \%)$ and $4(21 \%)$ had Lennox Gastaut syndrome. Pregabalin was maintained at $150-300 \mathrm{mg} /$ day. On pregabalin, one $(6 \%)$ child became completely seizure free and $7(37 \%)$ had $>50 \%$ seizure reduction. The percentage of children with daily seizure was reduced from $74 \%$ before pregabalin to $37 \%$ afterward $(p<0.002)$. Side effects were noted in $6(32 \%)$ children in the form of somnolence, weight gain, dizziness, and behavioral change. Most side effects were transient; however, the drug had to be withdrawn in $5(26 \%)$ children for of lack of efficacy and in $2(11 \%)$ for worsening of myoclonic epilepsy.

Pregabalin is a useful addition in the treatment of refractory childhood epilepsy. The drug should be used with caution in myoclonic epilepsy. Controlled studies are needed to establish the long term efficacy and tolerability in children.

Lecture IV:

\section{"A Substance in Broad Beans (Vicia faba) Protects from Convulsions" Dr. Mustafa Salih, KSU, Saudi Arabia}

Vicia faba (VF, broad beans) constitutes a major food item for the River Nile populations in Sudan and Egypt. Contrary to tropical Africa where the prevalence of epilepsy is 2-3 times that reported in industrialized countries, the prevalence rate of epilepsy (0.9-1 per 1000) among school children of Khartoum Province, Sudan is lower compared to Europe and North America.

To explore whether broad beans contained any anticonvulsant that can explain this observation, Balb/c 
mice were treated either with $\mathrm{VF}$ extract $(0.01 \mathrm{ml} / \mathrm{g})$ or kept as control. Various doses of strychnine and picrotoxin were used to explore the effect of VF extract on strychnine-sensitive glycine receptors (GlyRs) and $\mathrm{GABA}_{A}$ receptors. Diazepam (DIZ) was used as anticonvulsant. Thin layer chromatography was run for the extract against phenobartitone, DIZ and/or glycine. VF extract demonstrated a clear protective effect against strychnine-induced convulsions and death in Balb/c mice. Diazepam (20 mg/kg,1P), administered $20 \mathrm{~min}$ utes prior to strychnine $(0.112 \mathrm{mg} / \mathrm{kg}, 1 \mathrm{P})$ increased the survival rate to $66.7 \%$ and further to $100 \%$ when given with $\mathrm{VF}$ extract $(0.01 \mathrm{ml} / \mathrm{g})$. Various doses of DZ protected from picrotoxin-induced convulsions (PICS, $40 \mathrm{mg} / \mathrm{kg}, 1 \mathrm{P}$ ) and deaths. Pretreatment of mice with VF extract was not protective. On chromatography, VF extract separated in an identical manner to that of the glycine spot. The extract of broad beans (VF) protects against convulsions probably through the inhibitory GlyRs, and may contain a substance which is intimately related to glycine.

\section{Epilepsy Symposium: Part IV}

\section{Lecture I:}

\section{"The Ketogenic Diet: Update"}

Dr. Adel Mahmoud, KFMC, Saudi Arabia

As old as the history, the Ketogenic diet is a fasting as a treatment option for seizures was mentioned long before Christ. In modern times, Guelpa and Marie mentioned the effect of dietary manipulation on epilepsy as early as 1911 . The diet lost its popularity when effective antiepileptic drugs such as carbamazepine and Valproic acid came into use. It returned to the media in a T.V. program in the United States in 1994. The latter did injustice to other modalities of treatment; but increased the awareness about this form of treatment. This was followed by an increase in research on the effects of the diet.

Children with epilepsy should have access to such kind of treatment as it is of proven efficacy. The diet has high fat, low protein and very low carbohydrate content. This is meant to boost the brain with ketone bodies instead of sugar to fuel the brain. The latest randomized trial that ran in UK showed efficacy of $90 \%$ in $7 \%$ and more than $50 \%$ in $38 \%$ seizure reduction in children who were resistant to at least 2 properly used antiepileptic drugs. There are different types of Ketogenic diet; allowing the regimen to be less rigid. Here we throw light on the history, scientific basis, possible mechanisms of action, indications and contraindications of the diet and we briefly mention different types and the result of a study that compared two types of it.

\section{Lecture II: "Which Patient for Vagal Nerve Stimulation?" Dr. Dominique Parain, France}

Main VNS studies in adults have shown a mean seizure reduction of about $50 \%$ at 2 years, with $40 \sim 50 \%$ of patients showing $>50 \%$ reduction in seizure frequency. Results in pediatric studies have been more variable. Mean seizure reduction varies between 40 and $58 \%$. Percentages of patients showing $>50 \%$ reduction in seizure frequency vary between 37 and $75 \%$, with $5-18 \%$ of patients becoming seizurefree.

We describe a cohort of 50 infants, children, adolescents and young adults, prospectively followed, classified by epileptic syndromes and treated with VNS with at least 12 months follow-up. This cohort showed favorable outcome within 12 months which was sustained at 24 months: $68 \%(19 / 28)$ showing $>50 \%$ reduction in seizure frequency, including $14 \%$ (4/28) who became seizure-free. Our results are in the upper range of pediatric studies.

Why some young patients' series show better results than others? In mixing our data and the literature, it seems that the explanation maybe between the age of implantation, the duration of epilepsy and above all, the type of epileptic syndrome. We shall discuss mainly the efficiency of VNS in the following epileptic syndromes.

- Partial epilepsy (especially nocturnal frontal, central, bitemporal, dysplasia).

- Refractory infantile spasms.

- Myoclono-astatic syndrome.

- Refractory epilepsy absence.

- Lennox Gastaut syndrome.

- Pseudo Lennox Gastaut.

- Refractory JME.

\section{Lecture III:}

"Epilepsy Surgery in Intractable Childhood Seizures"

Dr. Abdulrahman Al-Sabbagh, KFMC, Saudi Arabia 
Monday, November 24, 2008

\section{Pediatric Neuroradiology}

Lecture I:

"Neuroimaging of Neonatal Encephalopathy"

Dr. Susan Blaser, Canada

\section{Lecture II:}

\section{"Imaging of Normal White Matter Myelination Milestones" \\ Dr. Sattam Lingawi, KAU, Saudi Arabia}

The normal process of brain maturation may be followed by observing the predictable course of white matter myelination. As myelination proceeds, the lipid content of white matter tracts increases, with a concomitant decrease in relative water content. These biochemical changes lead to shortening of the T1 and T2 relaxation times of myelinated white matter. Shortening of the T1 relaxation time leads to higher signal intensity on T1-weighted images, whereas shortening of the $\mathrm{T} 2$ relaxation time leads to lower signal intensity on T2-weighted images. Myelinated white matter will therefore appear as increased signal intensity on T1weighted images and as decreased signal intensity on T2-weighted images.

Before the age of 6 months, T1-weighted images are more sensitive to the detection of myelination changes than T2-weighted images. This is due in part to the fact that T2-weighted images of the immature brain (before the age of 6 months) have relatively poor gray/white matter distinction because the higher water content of unmyelinated white matter causes a relative increase in signal intensity that more closely matches that of gray matter rather than the lower signal intensity of myelinated white matter.

In general, brain maturation proceeds from central to peripheral, from inferior to superior, and from posterior to anterior. Myelination of white matter proceeds rostrally from the brain stem to cerebellum and cerebrum through the cerebellar and cerebral peduncles.

\section{Lecture III:}

\section{"Structural Imaging in Pediatric Epilepsy"}

Dr. Eman Bakhsh, RKH, Saudi Arabia

Radiological advances have resulted in a plethora of imaging techniques that can be used to unravel the complexities of child with epilepsy. However, the assessment of such a child involves 4 key stages:
- Recognition of epileptic seizure

- Classification of seizure type

- Identification of the epilepsy syndrome

- Identification of underlying aetiology

The aim of imaging studies is to identify epilepsy syndrome, underlying aetiology and facilitate prognosis. However, imaging is not required in all children with epilepsy. In those selected children for imaging it is broadly divided into structural and functional imaging. MR has revolutionized neuroimaging to provide the best non-invasive structural evaluation of the brain.

CT Role is considerably diminished and is used as complementary or supportive tool, and can be used as an initial method of excluding tumors. It is ideal to detect calcification.

The objective of this overview is to provide help for the specialist to make an informed decision regarding how to best image a child with epilepsy.

\section{Lecture IV:}

"Neuroimaging in Metabolic Disorders"

Dr. Susan Blaser, Canada

Inborn errors of metabolism are disorders in which an enzyme deficiency leads to a clinically significant block in a metabolic pathway. This blockage usually results in either an accumulation of substrate with damage induced by either storage or toxicity or in a deficiency of the product or metabolite. Rapid diagnosis and, when possible in treatable disorders, metabolic rescue is imperative to ameliorate neurologic sequelae or prevent death. The neurologist, therefore, needs to be aware of these disorders.

\section{Lecture V:}

"The Joubert Syndrome"

Dr. Bernard Maria, USA

Joubert syndrome (JS) is an autosomal recessive disorder that occurs in approximately 1 in 100,000 people. Though newborns with JS are hypotonic and have breathing irregularities, and older children have global developmental delay and apraxia, the diagnosis is often rarely made before 3 years of age. In 1996, we described the molar tooth sign that enables prenatal or neonatal diagnosis of JS with magnetic resonance imaging. The molar tooth sign is widely viewed as the hallmark radiological finding in JS and related disorders: thick and straight superior cerebellar peduncles, deep interpeduncular fossa, and absent/hypoplastic vermis. 
For over a decade, we have conducted detailed analyses of the clinical phenotype, neurobehavioral findings, and structural and functional neuroanatomical aspects of JS. Multiple genes identified in the condition account for approximately $40 \%$ of cases. One of the extraordinary brain malformations in JS is non-decussation of major tracts including cerebellar-cortical and corticalspinal projections, and probably cortical-bulbar tracts. We showed that non-decussation of the superior cerebellar peduncles accounts for narrowing of the brainstem isthmus and for two of the three components of the molar tooth sign. In fact, JS may well be the first human CNS malformation with non-decussation of major ascending and descending motor tracts, with preservation of sensory tract crossing. Moreover, subsets of patients with JS have associated renal cystic disease and retinal disease that may be caused by cilia dysfunction. Interestingly, cilia and their constituent proteins have recently been shown to be present on neurons, and thus we propose the hypothesis that cilia dysfunction in motor neurons accounts for axonal misguidance in CNS development in JS.

\section{Neurometabolic Disorders}

\section{Lecture I: \\ "Inherited Metabolic Diseases of the Nervous Sys- tem"}

Dr. Moeen Al Sayed, KFSH\&RC, Saudi Arabia

\section{Lecture II: \\ "Inherited Leukodystrophies in Saudi Patients" \\ Dr. Zuhair Al Hassnan, KFSH\&RC, Saudi Arabia}

Inherited leukodystophies comprise a group of genetically heterogeneous disorders that primarily affect the brain white matter. Defects in various organellerelated biochemical pathways are now known to be implicated in the pathogenesis of leukodystrophies. In addition, novel causative genes have been described in different forms of leukodystrophies. Understanding the role of these pathways and genes has expounded new mechanisms of the pathogenesis of white matter diseases.

In our population, the high consanguinity rate has been the major factor behind the higher incidence of rare autosomal recessive neurodevelopmental disorders. In some conditions, rare variants have been observed more frequently in our population. As an example, metachromatic leukodystrophy (MLD) secondary to the cofactor SAP-B deficiency has been observed more frequently than the classic MLD caused by arylsulfatase A deficiency. Moreover, novel genetic syndromes will likely continue to be described from our population. The large size of our consanguineous families paves the way for a fruitful homozygosity mapping for discovering novel genes. The autosomal recessive Pelizaeus-Merzbacher-like disease, caused by mutated GJA12 gene, is illustrative. Various other biochemical and molecular defects have also been observed and/or described in Saudi patients with inherited leukodystophies.

In this presentation, we review the clinical and molecular profiles of inherited leukodystrophies in a cohort of Saudi patients, which may shed light on the spectrum of this group of disorders in our population.

\section{Neuromuscular Disorders}

\section{Lecture I: \\ "Congenital Muscular Dystrophies" \\ Dr. Jiri Vajsar, Canada}

Muscular dystrophies (MD) are a genetically and phenotypically heterogeneous group of hereditary diseases characterized by progressive skeletal muscle weakness and wasting. The understanding of the molecular basis of the different MD types has evolved rapidly in recent years. The most common MD in children worldwide is Duchenne MD (DMD) due to mutations in $X p 21$ gene resulting in the deficiency of the sarcolemmal protein dystrophin. After the identification of the dystrophin gene, other genes encoding dystrophin-glycoprotein complex (DGC) and other cytoplasmic and extracellular proteins have been identified. To date, mutations in the different genes have been shown to cause many different MD types including limb-girdle MDs (LGMD), fascio-scapulo-humeral MD (FSHD), collagenopathies and congenital MDs (CMD).

CMDs typically present at birth or early infancy with hypotonia and muscle weakness and they are classified genetically and/or immunohistopathologically. Recognized types are: merosin-deficient CMD, collagenopathies, alpha-dystroglycanopathies, nuclear envelopathy $\mathrm{CMD}$ due to lamin $A / C$ mutations and CMD with rigid spine with mutations in selenoprotein $N$. There is wide variation in clinical severity within each type of CMD and marked clinical overlap between the CMD types and with other neuromuscular disorders including structural and metabolic myopathies. 
However, the genetic background of many MD forms is still not known, yet precise genetic classification is of utmost importance for the diagnosis, prognosis, genetic counseling and family planning. In CMDs, the genetic knowledge and insights into their pathogenesis may in the future enable disease-specific therapeutic interventions.

\section{Lecture II:}

"Inherited Anterior Horn Cell Disease and Muscle Dystrophy: The Saudi Experience",

Dr. Mohammed Jumah, NGH, Saudi Arabia

In Saudi Arabia and most of the Arab and Muslim world consanguineous marriages are commonly practiced. This has led to marked increase in the prevalence of inherited diseases, including neurological and diseases of the neuromuscular unit. Extraordinary breakthroughs in the molecular pathogenesis of neuromuscular diseases (NMD) have resulted in the rapid development of new diagnostic tools. This remarkable progress has introduced new diagnostic tests that have allowed for more sophisticated approaches for the diagnosis and evaluation of patients with presumed neuromuscular disorders (NMDs). In a population like ours the use of advanced technology should be geared towards the development of new diagnostic tests for preventive strategies, such as premarital testing and prenatal diagnosis of such disabling and fatal NMDs in addition to it's routine diagnostic indications.

In this presentation I will review a decade's worth of experience of the Neurogenetic laboratory at King Abdullah International Medical Research Center (KAIM$\mathrm{RC}$ ) in developing diagnostic tests for the most common inherited neuromuscular diseases in the Kingdom. The final results of the world's largest population based carrier testing for Spinal Muscular Atrophy will be presented.

The studies in this presentation are supported by: King Abdullah International Medical Research center (KAIMRC), Prince Salman Center for Disability Research (PSCDR), and King Abdulaziz City for Science and Technology (KACST).

\section{Lecture III:}

\section{"Neuromuscular Disorders from the Old World"} Dr. Mustafa Salih, KSU, Saudi Arabia

The present communication highlights the contribution of our Region in the identification of new neuromuscular disorders, or the discovery of the underlying genes, through multidisciplinary, regional and international collaborations. All of these are autosomal recessively inherited, reflecting the high rate of consanguinity in populations of Arab descent in North Africa and the Arabian Peninsula.

With regards to diseases of muscle, it has been established that the integrity of the cytoskeletal extracellular linkage, mediated by the dystrophin glycoprotein complex (DGC), is important for the muscle membrane stability, and loss or abnormality of certain components lead to a disruption of this linkage and cause various forms of MD.

"Adhalin" or alpha sarcoglycan, derived from the Arabic Word "Adhal" for muscle, is one of the DGC which has recently been implicated in the pathogenesis of severe childhood autosomal recessive muscular dystrophy (SCARMD). During the last two decades, (SCARMD) was identified as a unique form of MD with high prevalence in populations of Arab descent in Africa and the Middle East. Over the decade 1982-1993, it was found to be commoner (30\%) than Duchenne MD (25\%) and similar to congenital MD (30\%) at King Khalid University Hospital (KKUH) in Riyadh.

The paper reviews the clinical and molecular pathological features of SCARMD seen in Saudi Arabia and North Africa, a newly described form of MD associated with deficiency of a component of the DGC ( $\beta$ dystroglycan); and a novel mutation in a Saudi family with congenital MD due to partial deficiency of another component of the DGC (i.e. merosin or LAMA2).

\section{Workshop I: \\ Neuromuscular Workshop \\ Dr. Jiri Vajsar, Canada}

Tuesday, November 25, 2008

\section{Childhood Stroke}

\section{Lecture I:}

\section{"Stroke in Children with Sickle Cell Disease" Dr. Michael Dowling, USA}

Children with sickle cell disease are at risk of a number of neurologic complications including a greatly increased incidence of stroke or silent infarction, cerebral vasculopathy or moya-moya, headache, seizures, and progressive intellectual decline. Stroke is a significant cause of death and disability with clinical stroke oc- 
curring in $8-10 \%$ of children with sickle cell disease. An even larger percentage of children with sickle cell disease are subject to smaller areas of cerebral infarction, which occur in the absence of obvious neurologic symptoms and thus are termed "silent infarction". Silent infarctions occur in $35 \%$ of children with sickle cell disease, and far from being silent, are associated with significant cognitive impairment.

Children with sickle cell disease at high risk of stroke can by identified by transcranial Doppler ultrasound and a randomized controlled clinical trial (STOP) demonstrated that stroke can be prevented by initiation of chronic transfusion. Subsequent studies (STOP2) demonstrated that these transfusions must be continued indefinitely and other studies looking at alternatives to continued transfusion such as hydroxyurea (SWITCH) and the use of chronic transfusion in children with silent infarction (SITT) are underway.

Traditionally, the diagnostic evaluation of sickle cell disease patients with stroke ends with the identification of the abnormal hemoglobin. There are few identified risk factors for stroke in this patient population with previous stroke, acute chest syndrome, and sickle vasculopathy or moya-moya predisposing to stroke. Identification of additional risk factors could lead to improved risk stratification and possibly strategies to prevent stroke.

\section{Lecture II:}

\section{"Management of Cerebral Palsy: Update"}

Dr. Fatimah Al Zahra, RMC, Saudi Arabia

Cerebral palsy is a term used for persistent unchanging disorder of movement and posture due to lesion of developing brain. It is generally diagnosed before the age of 5 years. Cerebral palsy is not a single disorder, but a group of disorders with diverse implications for children and family.

There are many different causes, with wide range of manifestations, of motor disorders and various associated problems.

- There is significant association with prematurity and LBW and birth asphyxia

- Because each child with CP is different, individual assessment and treatment are essential

- Careful history and examination as well as observation to determine possible factors.

- Brain imaging should be undertaken to establish timing and possible causes.

- CP can be classified according to motor type, distribution and severity (using GMFCS).
- Co-morbidities, such as epilepsy are more common in certain types of $\mathrm{CP}$.

- Management is a team approach, involving range of health professionals and teachers, with input from the family of paramount importance, it involves management of general health problems, plus management of associated disabilities, and assessment of his capabilities.

- Hopefully newly used medication in CP cases, like Adenosine and recently stem cell transplant may help such patients with chronic disabilities. Family education and support is mandatory.

\section{Lecture III:}

"Neonatal Stroke"

Dr. Michael Dowling, USA

Stroke is one of the top ten killers of children. Of the survivors, up to $60 \%$ have neurologic deficits that will persist for their lifetime, during which recurrent stroke will strike 10-25\%. Improvements in awareness and imaging, as well as the increasing survival of children with conditions which place them at risk for stroke, such as sickle cell disease, congenital heart disease, and cancer, have led to an increase incidence of arterial ischemic stroke (AIS) and cerebrosinovenous thrombosis (CSVT) to the range of 2.3-13 per 100,000 per year in children, with stroke in neonates occurring in 1 in 4000 live births.

Stroke is as common as childhood leukemia or brain tumors, yet unlike these disorders, until recently, there were no clinical guidelines, systematic research programs, or randomized clinical trials for stroke intervention or prevention in children. There are now three published guidelines for the diagnosis and management of stroke in children.

The International Pediatric Stroke Study is a collaborative effort of over 200 physicians at over 60 centers is expanding our understanding of the clinical presentations, etiologies and treatment options available around the world. We have established a multidisciplinary Pediatric Acute Stroke Team to aid in the identification and treatment of children with stroke. There are now multiple ongoing and planned investigations into the classification of pediatric stroke, the role of infection in cerebral vasculopathy and stroke, the safety of tPA in children, and the early prediction of motor outcomes through the detection of Wallerian degeneration of the corticospinal tract in the acute setting. 


\section{Lecture IV:}

"Nervous System Tumors"

Dr. Bernard Maria, USA

Tumors of the central nervous system (CNS) represent the most common solid tumors in children and with leukemia, account for more deaths in childhood than from any other diseases. In addition, children with neurofibromatosis type 1 (NF1), the most common neurocutaneous disorder, have a $10 \%$ lifetime risk of developing treatrment-resistant and malignant peripheral nervous system tumors (MPNSTs). The 2007 Neurobiology of Disease in Children (NDC) symposium (www.neurobiologyofdisease.com) held in Quebec City, Canada reviewed our current understanding of pediatric CNS tumors and proposed new research priorities. The proceedings of the meeting were published in the October 2008 issue of the Journal of Child Neurology (Volume 23, Number 10).

I will review highlights of the NDC conference and discuss progress in developing a novel therapy for CNS tumors (malignant gliomas) and peripheral nervous system (PNS) tumors (MPNSTs). Hyaluronan is a large polymeric glycosaminoglycan that is abundantly secreted by malignant CNS and PNS tumors. Hyaluronan and its primary receptor CD44 are present on the surface of glioma cells, MPNST cells, and highly treatment-treatment resistant glioma stem-like cells. Hyaluronan-CD44 interactions constitutively co-regulate activation of receptor tyrosine kinases, membrane drug pumps enriched in stem-like cells, anti-apoptotic activities, invasiveness, and resistance to chemotherapy and radiotherapy. We found that hyaluronan antagonism via treatment with oligomers (o-HA) downregulates malignant behaviors in vitro and in vivo. I will describe the steps needed to translate our basic science and preclinical trial findings into a novel therapy for children and adults with malignant CNS and PNS tumors.

\section{Lecture V:}

"Double-blind randomized study of using Levetiracetam in the treatment of tics in Tourette syndrome"

\section{Dr. Yasser Awaad, KFMC, Saudi Arabia}

Some drugs currently used to treat tics have drawbacks, including the risk of side effects such as tardive dyskinesia. Therapeutic options with better safety profiles are needed. Levetiracetam is an anti epileptic drug with atypical GABAergic effects that might be beneficial for this indication.
To evaluate the effects of levetiracetam on motor and focal tics, behavior, and school performance in children and adolescents with tics and Tourette syndrome.

24 patients, age: $=; 18$ years, with tics and Tourette syndrome were enrolled in this prospective, doubleblinded placebo randomized study for 8 weeks. Each group had 12 patients. The initial starting dose of levetiracetam was $250 \mathrm{mg} / \mathrm{d}$. The dosage was titrated over 3 weeks to 1,000 to $2,000 \mathrm{mg} / \mathrm{d}$. Clinical outcomes were assessed with the Clinical Global Impression Scale, Yale Global Tic Severity Scale, and Revised Conners' Scale.

10 out of 12 patients in the Levetiracetam group showed improvements based on all of the scales used and 4 patients improved with regard to behavior and school performance. 2 patients dropped out. 9 patients out of 12 patients in the placebo group showed no improvement, one patient showed a great placebo effect, and 2 patients dropped out of the study. Levetiracetam was generally well tolerated. 2 patients discontinued because of exaggeration of pre-existing behavioral problems.

Levetiracetam may be useful in treating tics in children and adolescents. Given its established safety profile, levetiracetam is a candidate for additional evaluation.

\section{Movement Disorders}

\section{Workshop I:}

Workshop on Movement Disorders

Dr. Yasser Awaad, KFMC, Saudi Arabia

Lecture I:

"Chronic Childhood Ataxia"

Dr. Raidah Al Baradaei, King Faisal University Hospital, Saudi Arabia

Ataxia is defined as an inability to maintain normal posture and smoothness of movement. Neurologic symptoms and signs may accompany ataxia. Consequently, many variations are encountered in the clinical phenotype, ranging from findings of pure cerebellar dysfunction to mixed patterns of involvement reflecting extrapyramidal pathways, brainstem, and cerebral cortical involvement. A wide range of molecular defects have been identified in which the spinocerebellar pathways are involved.

Despite this remarkable diversity of genetic defects and mechanisms, the pathologic responses within the 
nervous system are limited in terms of the targeted pathways. This feature likely contributes to significant overlap seen in the clinical presentation. Nevertheless, delineation of the clinical phenotype represents an important first step in the diagnostic process. The clinical phenotype guides the geneticist in a search for appropriate diagnostic tests, reducing costs of laboratory work.

This presentation will focus about a case presentation of child with chronic ataxia, then there will be a review about the understanding of inherited neurologic and metabolic disorders manifesting with ataxia as a clinical features, laboratory findings, and pathophysiologic insights gleaned from molecular genetic studies, as well as current treatment strategies in management.

\section{Lecture II:}

"Clinical Approach to Neurogenetics of Movement Disorders; Illustrating Cases"

\section{Dr. Saeed Boholega, KFSH\&RC, Saudi Arabia}

Movement disorders are a clinically and genetically heterogeneous group of neurological conditions sharing several common features, such as impaired control and/or execution of movements or presence of involuntary movements. There may be considerable phenotypic overlap between different movement disorders in individual patients or co-occurrence of more than one movement disorder in the same individual. For example, early-onset Parkinson's Disease (PD) patients frequently also suffer from dystonia; conversely, patients with dopa-responsive dystonia (DRD) may present with isolated parkinsonism. Patients with spinocerebellar ataxia (SCA) 17 may show dystonia as presenting sign, and PD and RLS sometimes coexist. Interestingly, a link between different movement disorders has recently also been described at the molecular level.

For example, torsinA, the protein encoded by the DYT1 dystonia gene, is a component of Lewy bodies in PD patients and co-localizes with alpha-synculcein (SNCA) immunopositive inclusions. Further, overexpression of torsinA and specific heat shock proteins suppressed SNCA aggregation in this cellular model, whereas mutant torsinA had no effect. Linking SCA and Dystonia, intranuclear neuronal inclusions were found to be torsinQ-immunoreactive in SCA3 cases. Due to common neurotransmitters and neuronal pathways, movement disorders are frequently associated with psychiatric abnormalities. Examples include hallucinations in PD or obsessive compulsive disorder in myoclonus-dystonia (M-D). Overall, movement disor- ders are a relatively frequent finding in the general population and are mostly associated with severe disability and high treatment costs, thus markedly reducing quality of life of the patients and their families, as well as posing a considerable economic burden on health care systems.

\section{Child Psychiatry}

\section{Lecture I:}

"Coping with Chronic Illness - How do Kids do it?" Dr. Nihal Erfan KFMC, Saudi Arabia

The true understanding of the chronicity of an illness only hits home when it affects not only the patient but the entire family. When a young child has a chronic illness, it is crucial to take into account how this will affect the child's development, whether physical, emotional or cognitive from that point on. It is also important to take into consideration where this child or adolescent is in terms of their development to then predict outcome. Many variables related to the specific chronic illness will also definitely affect outcome, things like severity of the illness, morbidity and mortality of the illness and treatment modalities are some to consider. Also many variables related to the individual patient will affect outcome. The cognitive development and how concepts are formed in children will be highlighted. Then the psychiatric and psychological sequelae of a chronic illness will be reviewed. This is to better identify those patients at greater risk of developing a psychiatric disorder secondary to their illness and hence, to be able to provide better support and more efficient treatment

\section{Lecture II:}

"Attention Deficit Hyperactivity Disorder"

Dr. Suad Yamani, KFSH\&RC, Saudi Arabia

\section{Lecture III:}

\section{"Childhood Learning Disabilities"}

Dr. Cheryl Oandasan, Prince Sultan Bin Abdulaziz Humanitarian City, Saudi Arabia

Among children with disability in Saudi Arabia, the prevalence of intellectual disability (i.e. learning disability, mental retardation, developmental disability) has been estimated to be $51.3 \%$ (Country Profile on Disability -KSA, 2002). Often children with moderate to severe learning disabilities have the added challenge of comorbid conditions such as physical or sen- 
sory disabilities and epilepsy. Behavioural and psychiatric problems are also over-represented in this population of children. This paper will outline a practical approach for pediatric neurologists to understand and manage challenging behaviours of children with learning disabilities such as temper tantrums, aggression, sleep disorders and self injurious behaviour. 